\title{
Challenges of Knowledge Management for Disaster Risk Reduction in Iran
}

\author{
Navab Shamspour ${ }^{1,5} \mathbb{D}$, Esmaeil Kavousi $^{2} \mathbb{D}$, Mojtaba Moazzami $^{3} \mathbb{D}$, Abbasali Ghaiyoomi $^{4} \mathbb{D}$, \\ Milad Ahmadi Marzaleh ${ }^{5,6,7,8} \mathbb{D}$
}

Date of submission: 10 Oct. 2020 Date of acceptance: 09 Nov. 2020

\begin{abstract}
INTRODUCTION: Knowledge is a powerful resource to help governments, organizations, and communities to plan and improve their performance in mitigating the harmful effects of disasters. There is a crucial need for knowledge in all phases of disaster risk management, namely prevention, risk reduction, preparedness, as well as response and recovery. The present study aimed to assess the organizational, discourse, and metaphorical challenges and knowledge management improvement policies in disaster risk management in Iran.

METHODS: This qualitative study was conducted in Iran in 2016. The needed data were collected through in-depth interviews with 21 experts and policymakers and analyzed by content analysis method.

FINDINGS: Based on the obtained results, the following five categories were extracted: 1 . current problems, 2. organizational and structural problems, 3. discourse level, 4. level of metaphors, and 5. knowledge management improvement policies in the disaster response phase in Iran.

CONCLUSION: As evidenced by the obtained results, the challenges presented to knowledge management in disasters included the repetition of the same problems of previous disasters, non-use of the experiences of previous disasters, forgetting disasters, the long return period of some disasters, failure to transfer field and operational experiences, ignoring the bitter experiences of disasters, violating processes and sometimes copying without considering operational experiences, lack of expert documentarians or disaster documentation teams, as well as too many disasters and too little documentation. Moreover, it was found that these challenges are rooted in structural, cultural, social, discourse, and mythological problems.
\end{abstract}

Original Article

Keywords: Culture; Disasters and Hazards; Documentation; Knowledge Management.

How to cite this article: Shamspour N, Kavousi E, Moazzami M, Ghaiyoomi A, Ahmadi Marzaleh M. Challenges of Knowledge Management for Disaster Risk Reduction in Iran. Sci J Rescue Relief 2020; 12(3): 210-216.

\section{Introduction}

$\mathrm{D}$ isasters can affect communities in numerous ways, harming humans, the environment, and infrastructures.
Nonetheless, some management measures, such as national and local capacity building, planning, and training, can reduce vulnerability to disaster events (1). Almost all countries are prone to natural

\footnotetext{
1-PhD Student, Educational Management, Department of Educational Management, Faculty of Management, Islamic Azad University, North Tehran Branch, Tehran, Iran

2-PhD, Department of Cultural Management, Faculty of Management, Islamic Azad university, North Tehran Branch, Tehran, Iran

3-PhD, Department of Higher Education Management, Faculty of Management, Islamic Azad University, North Tehran Branch, Tehran, Iran

4-PhD, Department of Cultural Management, Faculty of Management, Islamic Azad University, North Tehran Branch, Tehran, Iran.

5- Research Center for Emergency and Disaster Resilience, Red Crescent Society of the Islamic Republic of Iran, Tehran, Iran

6- PhD, Applied Science Higher Education Institute, Red Crescent Society of the Islamic Republic of Iran, Tehran, Iran

7-PhD, Disaster and Emergency Health, School of Management and Medical Information, Shiraz University of Medical Sciences, Shiraz, Iran

8-Master of Public Health, Health Policy Research Center, Shiraz University of Medical Sciences, Shiraz, Iran

Correspondence to: Esmaeil Kavousi, Email: ekavousi@gmail.com
} 
disasters, such as hurricanes, floods, earthquakes, fires, famines, terrorist attacks, volcanic eruptions, chemical disasters, and diseases. Natural disasters may start quickly or gradually; however, they can exert adverse effects on the health of people, society, and the economy (2).

Iran is also exposed to a wide range of natural hazards. Among the most important natural and man-made hazards in this country, we can refer to earthquakes, floods, droughts, and traffic accidents (3). The annual number of disasters has increased over the past decade and affected numerous people $(4,5)$. Nevertheless, we are witnessing the occurrence of natural and manmade disasters with all their negative consequences which seriously affect millions of people across the globe every year (6). Although the negative consequences of disasters cannot be reversed, efficient efforts can minimize their destructive effects. There is no doubt that knowledge of disaster management strategies, efficient strategies, and lessons learned, as well as risk reduction measures and preparedness planning, can be of great help for mitigating these harmful effects (7).

These measures and awareness bring about positive effects, such as research, documentation of experiences, and knowledge management in times of crisis. They are the requirements for a resilient society and can be used for the prevention and mitigation of future crises and better preparedness to deal with them. Moreover, relief organizations need the following five skills: a systematic approach to problem-solving, trying out new solutions, learning from past experiences, learning from others' experiences, and effective transfer of learning in the organization. In addition, those involved in disaster management need to improve their skills and raise their level of knowledge.

To attain these goals, it is necessary to invest in systems, databases, and network structures to create a lessons-learned culture and adopt the most efficient methods. Furthermore, some studies indicated that community vulnerability is significantly associated with deficiencies in knowledge management $(8,9)$. However, there is a widely accepted need for disaster risk management in the face of diverse natural hazards. In this regard, knowledge management, along with key measures, lessons learned, and effective knowledge transfer, can support this management which depends on key institutional, cultural, and social factors and needs to be identified in different societies.

In light of the aforementioned issues, the present study aimed to identify the problems and challenges presented to knowledge management for disaster risk reduction at institutional, cultural, discourse, and metaphorical levels in Iran.

\section{Methods}

This qualitative study was conducted using a content analysis technique in cyberspace within July-September 2020. The needed data were collected through in-depth virtual interviews with experts and policymakers. The inclusion criteria entailed: 1. a minimum of two years of work experience in the field of crisis management, 2. a minimum of master's degree education. On the other hand, the exclusion criteria included: 1. mental and psychological unpreparedness to participate in the study, 2. unwillingness to participate.

The purposive and snowball sampling method was used in the current study. This signifies that each specialist introduced the next specialist and finally all the members formed a group. Thereafter, the items were asked, answered, and discussed by the experts. Each of the experts was asked deep and semi-structured questions about problems at organizational, discourse and metaphor levels, as well as improvement of knowledge management policy making in the disaster response phase in Iran.

The questions included:

1. What problems are presented to knowledge management in the disaster response phase in Iran?

2. What is Knowledge management discourse in the disaster response phase in Iran?

3. What is the organizational level of knowledge management in the disaster response phase in Iran?

4. What policies can be adopted to improve knowledge management in the disaster response phase in Iran?

Tracer questions were also asked based on participants' responses. Each question needed to be answered in one week in a Telegram group. Interviews continued until data saturation; moreover, authentic reports were also analyzed. Upon the completion of interviews and revision of reports, the interviews were transcribed. 
Subsequently, problems, organizational, discourse and metaphor levels, as well as improvement policies were extracted, and data analysis was performed manually. The thematic analysis method was used to analyze the interviews (10). This method follows a six-step process:

Familiarize yourself with your data, assigning preliminary codes to your data in order to describe the content, searching for patterns or themes in your codes across the different interviews, reviewing themes, defining and naming themes, and producing your report (11). After analyzing interview texts, 60 codes were finally extracted, which were assigned to 5 groups by the removal and reanalysis of duplicate codes.

Rigor: Lincoln and Guba's criteria were used to ensure the accuracy of qualitative data (9). Moreover, trustworthiness, transformability, dependability, and confirmability were assessed. Ethical considerations were as follows: 1 . ensuring the confidentiality of personal information 2 . providing research information to participants, 3 . obtaining written consent from experts 4 . and appreciating all participants.

\section{Findings}

Out of 21 participants, 19 cases were male, and the mean age of the experts was 46 years. The demographic characteristics of the interviewed experts are displayed in Table 1.

Table 2 presents the categories and subcategories resulting from interviews and analysis of reports on knowledge management in the disaster response phase in Iran.

One disaster management expert said: "Repetition of the same mistakes in disasters is a serious problem. For instance, the problems encountered in 1990 Manjil-Rudbar earthquake were repeated in Mormori earthquake" (P.4). A Google search provides about 33,400 articles on
Bam earthquake in English. The same search results in 47,900 cases in Persian. Google Scholar search retrieves about 1,490 scientific articles and expert and research reports related to Bam earthquake published in various journals since the earthquake.

As a comparison, we can obtain 5,110 articles by searching for the Indian Ocean tsunami of 2004 which occurred exactly one year after Bam earthquake. Moreover, if we conduct the same research for the 2005 Hurricane in the United States, we can retrieve 40,900 research articles and reports. Although not all studies in these systems can be traced, the majority of them are listed in this search engine. This preliminary study demonstrates that crises in our country have been scarcely researched and documented. Google Scholar search of Bam earthquake retrieved 140 cases in 2004 which gradually increased to 217 cases by 2008 .

This trend has been declining since 2009, reaching 152 cases in 2010. If we perform the same study in the databases of reputable international journals, we will find a smaller number of articles. For example, a query which was conducted on the Scholar Portal yielded around 121 records, 98 of which were research articles. These articles have been published in 60 journals in various scientific fields; moreover, several articles have also been published in Persian-language journals, mostly in the field of medical sciences. A cursory glance at the titles of the articles shows that majority of them are about engineering and science, and other papers were published in other scientific fields. Nonetheless, there is a dearth of articles in the fields of crisis management, humanities, and social sciences. This indicates that research in disaster events has not been adequately organized in Iran.

Table 1. Demographic characteristics of the interviewed experts

\begin{tabular}{|c|c|c|c|c|c|c|c|}
\hline \multirow{2}{*}{ Row } & \multirow{2}{*}{ Specialty / Field } & \multirow{2}{*}{$\mathbf{n}$} & \multirow{2}{*}{ Mean age (year) } & \multicolumn{2}{|c|}{ Gender } & \multicolumn{2}{|c|}{ Education } \\
\hline & & & & Male & Female & Maters' degree & PhD \\
\hline 1 & Crisis management & 12 & 42 & 11 & 1 & 10 & 2 \\
\hline 2 & Education Management & 4 & 41 & 3 & 1 & 3 & 1 \\
\hline 3 & Sociology & 2 & 45 & 2 & 0 & 2 & \\
\hline 4 & Cultural management & 1 & 42 & 0 & 1 & 1 & \\
\hline 5 & Psychology & 1 & 51 & 1 & 0 & 1 & \\
\hline 6 & Medicine & 3 & 49 & 3 & & & 3 \\
\hline 7 & Total & 1 & 45 & 19 & 3 & & \\
\hline
\end{tabular}


satisfactory in other areas of documentation, such as reports, news, photos, lessons learned, and narrations. Despite the highest number of disasters in Iran, there is a scarcity of information, statistics, and documents in this regard. One expert in knowledge management improvement policies said: "To create a standard structure, all activities need to be standardized, and all

Table 2. Categories and subcategories resulting from interviews and analysis of reports on knowledge management in the disaster response phase in Iran

\begin{tabular}{|c|c|}
\hline Category & Subcategory \\
\hline $\begin{array}{l}\text { Current } \\
\text { problems }\end{array}$ & $\begin{array}{c}\text { Repeating the problems of previous disasters } \\
\text { Lack of documentation and experiences of previous disasters } \\
\text { Forgetting the disasters } \\
\text { Long return period of some disasters } \\
\text { Lack of transfer of field and operational experiences } \\
\text { Not learning from the bitter experiences of previous disasters } \\
\text { Violating processes and sometimes copying without considering operational experiences } \\
\text { Lack of expert documentarians or disaster documentation teams } \\
\text { Too many disasters and too little documentation }\end{array}$ \\
\hline $\begin{array}{l}\text { Organizational } \\
\text { and structural } \\
\text { problems }\end{array}$ & $\begin{array}{l}\text { Interim Management } \\
\text { Lack or shortage of specialized knowledge of the assigned managerial task } \\
\text { The dominance of a work culture emphasizing fast-paying activities } \\
\text { Dominance of the culture of admiring show-off activities in organizations } \\
\text { Ignoring the future in relief and operational organizations } \\
\text { Pritorozing political and group interests over organizational interests } \\
\text { Employees' unfamiliarity with documentation } \\
\text { Lack of standards, as well as specific and practical software for information registration } \\
\text { Familiarity with effective methods of documenting experiences } \\
\text { Lack of relatively accurate criteria for evaluating and selecting valuable experiences } \\
\text { Not reprimanding operational managers } \\
\text { Withholding experience from new managers } \\
\text { Lack of documentation instructions, especially in the operations section }\end{array}$ \\
\hline $\begin{array}{l}\text { Discourse } \\
\text { level }\end{array}$ & $\begin{array}{l}\text { Informal organizations that support managers and hinder the provision of transparent reports } \\
\text { Fear of reprimand and media rumors due to transparent and accurate reporting } \\
\text { Political rumors } \\
\text { Absence of critical discourse } \\
\text { Lack of communication between the university and operational organizations } \\
\text { Lack of reference systems for evaluation and analysis } \\
\text { Emotional discourse and forgetting disasters } \\
\text { Individual monopoly on documentation of previous experiences } \\
\text { Lack of discourse to analyze and refine experiences } \\
\text { Failure to hold analysis sessions after operations } \\
\text { Absence of researchers in the field of operations and communication with operational teams } \\
\text { Failure to publish national and international reports of relief organizations }\end{array}$ \\
\hline $\begin{array}{l}\text { Level of } \\
\text { metaphors }\end{array}$ & $\begin{array}{c}\text { Imitation training system } \\
\text { Intrinsic reluctance to write } \\
\text { Disbelief in a historical review } \\
\text { Omniscient point of view } \\
\text { Adoption of a passive system against the threats of disasters }\end{array}$ \\
\hline $\begin{array}{l}\text { Improvement } \\
\text { policies }\end{array}$ & $\begin{array}{c}\text { Creating organizational structure } \\
\text { Training managers and employees with documentation methods } \\
\text { Using documentation to redesign }\end{array}$ \\
\hline
\end{tabular}


authorities must have public acceptance and be accountable in some way" (P.2). Nonetheless, natural and man-made disasters occur with all their negative consequences; however, each disaster also brings some positive effects.

Disasters can reveal hidden wounds. Furthermore, research and documentation of crisis experiences are their positive effects that are the requirements for a resilient society and can be used for the prevention and mitigation of future crises and better preparedness to deal with them. Studies have demonstrated that numerous societies found themselves in a desirable situation after the crisis. The most important reason for this improvement was the correction of deficiencies. Field research is one of the best and most effective ways to answer numerous questions about disasters, people's behavior, accountability of responsible organizations, and documenting post-disaster lessons learned.

One expert on knowledge management metaphors said: "We do not believe in writing, despite the fact that Islamic hadiths emphasized that we acquire knowledge by writing" (P.7). Another expert on discourse level said: "When a disaster occurs, it is a heated discussion in all media (radio, television, newspapers, magazines, and websites); nonetheless, just a month later, it will lose its significance" (P.8). Rescue and relief operations in the response phase bear a close resemblance to defense and military operations.

Research on post-disaster reports highlights the necessity of preparing reports during and after operations, as well as lessons learned, in natural disasters, such as floods, earthquakes, major storms (such as hurricanes and tsunamis), as well as man-made disasters, such as nuclear and chemical hazards, terrorism, explosions, and large fires. These reports can be of great help for mitigating the fatalities and casualties of disaster events. The lessons learned are positive or negative knowledge and experience gained from real events. They are suggestive of our future potentials and capabilities, and emphasis on strengths and weaknesses and provision of efficient problem detection strategies are the most pronounced outcomes of knowledge management. Furthermore, continuous field reports and post-operation analysis play a key role in the improvement of operational situations.

\section{Discussion and Conclusion}

Knowledge management has attracted assiduous attention over the last decade. Numerous companies and organizations have used knowledge management tools, such as e-learning, focus groups, document management devices, and other policies to motivate employees and share their knowledge. The implementation of an effective knowledge management strategy is a prerequisite for the success of organizations and societies in the era known as knowledge-based economy. Knowledge signifies a complex flow of structured experiences, values, background information, and expert insights that provide a framework for evaluating and integrating new experiences and information originating from individuals' perspectives.

In organizations and societies, knowledge can be found not only in documents and resources but also in everyday work, processes, practices, and norms. Therefore, crisis management is one of the critical needs of today's society. In order to properly deal with disasters and respond to them according to international standards, it is necessary to manage crisis management knowledge and institutionalize it in scientific and executive systems. Today, this knowledge is recognized as one of the most important branches of crisis and disaster management all across the globe (12).

As evidenced by the obtained results, the challenges presented to knowledge management in disasters included the repetition of the problems of previous disasters, non-use of the experiences of previous disasters, forgetting disasters, the long return period of some disasters, failure to transfer field and operational experiences, ignoring the bitter experiences of disasters, violating processes and sometimes copying without considering operational experiences, lack of expert documentarians or disaster documentation teams, as well as too many disasters and too little documentation. Moreover, it was found that these challenges are rooted in structural, cultural, social, discourse, and mythological problems.

At the structural and organizational level, some of the challenges which need consideration for successful implementation of knowledge management in disaster risk management were as follows: temporary management concept, absence or shortage of specialized knowledge of the assigned managerial task, The dominance of a 
work culture emphasizing fast-paying activities, neglecting the future in relief and operational organizations, Prioritization of political, partisan, and group interests over organizational interests, employees' unfamiliarity with documentation, lack of standards, as well as specific and practical software for information registration, unfamiliarity with effective methods of experience documentations, lack of relatively accurate criteria to evaluate and select valuable experiences, not reprimanding operational managers, withholding experiences from new managers, lack of documentation guidelines, especially in the operational sector.

Furthermore, the following issues should be taken into account: the establishment of the National Crisis Organization at the macro level for operations, establishment of a deputy in the Crisis Management Organization, comprehensive documentation of operations (an information system responsible for the provision, retrieval, analysis, and proper use of information in crisis management), documenting the experiences of managers and operational forces through qualitative research, such as autobiography, action research, phenomenology, documenting organizational documents and activities by setting up a documentation unit in all operational organizations (the use of effective data registration software and evaluating data quality, as well as data collection and management and turning it into useful information, form the basis of a successful planning in all stages before, during, and after the operation), the implementation of research projects to develop guidelines, checklists, and all components of operation documentation from needs assessment up to completion, the preparation of documentation guidelines, holding documentation courses, taking successful organizations in the field of documentation as a role model, as well as the evaluation of documentation software and methods based on existing scenarios.

In addition, the problems and challenges that need to be addressed in the underlying layers are as follows: informal organizations that support managers and hinder the provision of transparent reports, fear of reprimand and media rumors due to transparent and accurate reporting, political rumors, absence of critical discourse, lack of communication between the university and operational organizations, lack of reference systems for evaluation and analysis, forgetting disasters, individual monopoly on documentation of previous experiences, lack of discourse to analyze and refine experiences, failure to hold analysis sessions after the operations, absence of researchers in the field of operations and communication with operational teams, failure to publish national and international reports of relief organizations, imitation training system, intrinsic reluctance to write, disbelief in historical review, omniscient point of view, and adoption of a passive system against the threats of disasters.

To address these challenges, it is suggested that uncertainties be identified in a prospective study, and some scenarios be considered for the successful implementation of knowledge management in crisis management organizations. It is evident that disasters cannot be eradicated from the face of the earth; nonetheless, effective management and response can save thousands of lives. The increasing rate of natural disasters in the last decade highlights the need for more attention to disaster management on the part of the research community. According to the literature, disaster risk management is an interdisciplinary issue; therefore, researchers in various fields should collaborate to help advance disaster management research. A comprehensive and reliable knowledge base in which we share and reuse knowledge can make a great contribution to the reduction of damages and losses that could arise from disasters.

\section{Acknowledgments}

None.

\section{Conflict of Interests}

The authors have no conflict of interest regarding the publication of the present article.

\section{Footnotes}

Informed consent: Informed consent was obtained from all participants in the study.

Funding/Support: Nil.

Financial Disclosure: None declared.

\section{References}

1. Unisdr U. Hyogo framework for action 2005-2015: building the resilience of nations and communities to disasters. Extract from the final report of the World Conference on Disaster Reduction (A/CONF 
206/6). Geneva: The United Nations International Strategy for Disaster Reduction; 2005.

2. Guha-Sapir D, Vos F, Below R, Ponserre S. Annual disaster statistical review 2011: the numbers and trends. Brussels, Belgium: Centre for Research on the Epidemiology of Disasters (CRED); 2012.

3. Ardalan A, Mowafi H, Khoshsabeghe Y. Impacts of natural hazards on primary health care facilities of Iran: a 10-year retrospective survey. PLoS Curr 2013; 5: 12.

4. Azami-Aghdash S, Gorji HA, Sadeghi-Bazargani $\mathrm{H}$, Shabaninejad H. Epidemiology of road traffic injuries in Iran: based on the data from Disaster Management Information System (DMIS) of the Iranian Red Crescent. Iran Red Crescent Med J 2017; 19(1): e38743.

5. Jahanbakhsh $\mathrm{M}$, Tavakoli $\mathrm{N}$, Hadadpour A. Designing disaster victims'medical record; a step toward crisis management. Health Inform Manag 2011; 7(4): 400-9.

6. Ardalan A, Kandi M, Osooli M, Shamseddini A. Profile of natural hazards in I.R.Iran, 1970-2010. Tehran: Project of Iran's National Institute of Health Research; 2012.
7. Ager A, Baillie Smith M, Barbelet V, Carpenter S, Carter W, Cartwright A, et al. World disasters report: focus on local actors, the key to humanitarian effectiveness. Geneva, Switzerland: The International Federation of Red Cross and Red Crescent Societies; 2015.

8. Moe TL, Gehbauer F, Senitz S, Mueller M. Balanced scorecard for natural disaster management projects. Disast Prev Manag Int J 2007; 16(5): 785-806.

9. Seneviratne TK, Amaratunga D, Haigh R, Pathirage $\mathrm{CP}$. Knowledge management for disaster resilience: Identification of key success factors. Manchester, England: University of Salford; 2010.

10. Braun V, Clarke V. Using thematic analysis in psychology. Qualit Res Psychol 2006; 3(2): 77-101.

11. Schwandt TA, Lincoln YS, Guba EG. Judging interpretations: but is it rigorous? Trustworthiness and authenticity in naturalistic evaluation. N Direct Evaluat 2007; 2007(114):11-25.

12. Zhang D, Zhou L, Nunamaker Jr JF. A knowledge management framework for the support of decision making in humanitarian assistance/disaster relief. Knowl Inform Syst 2002; 4(3): 370-85. 